\title{
Ultrasonic speckle correlation imaging of 2D particle velocity profiles in multiphase flows
}

\author{
J. Carlson ${ }^{\mathrm{a}, *}$, R.K. Ing ${ }^{\mathrm{b}}$ \\ ${ }^{a}$ EISLAB, University of Technology, Department of Computer Science and Electrical Engineering, SE-971 87 Lulea, Sweden \\ ${ }^{\mathrm{b}}$ Laboratoire Ondes et Acoustique, ESPCI, 10 rue Vauquelin, FR-75231 Paris, Cedex 05, France
}

\begin{abstract}
Two-dimensional ultrasonic speckle correlation velocimetry (USV) is a new technique that allows imaging of moving scattering media, at a high frame-rate. In this paper we apply the technique to determine two-dimensional particle velocity profiles of multi-

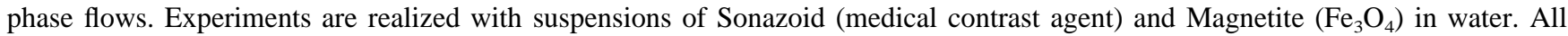
measurements are performed in a vertical pipe with the flow moving downwards. The two-dimensional particle velocity profiles are then compared with a reference liquid volume flow velocity. As expected from theory, the heavier Magnetite particles have slightly higher velocity than the liquid, whereas the contrast agent simply follows the liquid motion.

The proposed technique can be used in combination with other techniques to measure the mass flow of the solid phase, in solid/liquid multiphase flow. This is generally more interesting than measuring the bulk mass or volume flow.

(C) 2003 Elsevier Ltd. All rights reserved.
\end{abstract}

Keywords: Ultrasound; Multiphase flow; Speckle correlation imaging; Velocity measurements

\section{Introduction}

During the last $10-15$ years, the measurement of different properties of multiphase flows has received much attention in the flow measurement community. In some industrial processes, such as the mining industry, a liquid flow is used to transport solid particles. In such flows, the major interest lies in estimating the amount of particles transported, and not in measuring the bulk flow itself. Ultrasound provides a direct way of global and noninvasive measurement of several parameters of such flows. For single-phase flows of liquids and gases there are several well-established ultrasonic techniques available, based on the differences in transit-time for sound propagating upstream and downstream in the flow. In the presence of particles, the sound is scattered and the waveform of the sound pulse is heavily distorted. Because of this, determining transit times becomes difficult, and therefore these techniques are not suitable for solid/liquid multiphase flows. Another technique is the ultrasonic Doppler velocimetry (UDV), where the flow

\footnotetext{
* Corresponding author.

E-mail address: Johan.Carlson@sm.luth.se (J. Carlson).
}

velocity is measured by estimating a Doppler shift of the transmitted sound wave, caused by the flow. Techniques called pulsed ultrasonic Doppler velocimetry are also used. This is unfortunately a somewhat misleading name, since instead of measuring the actual Doppler shift, the velocity is often determined by estimating the time delay between two backscattered pulses resulting from two consecutive transmissions. Contrary to the transit-time techniques, the use of pulsed UDV is limited to applications where the medium contains scatterers. For multiphase flows, cross-correlation methods are still often preferred over transit-time techniques. The most important reason for this is that transit-time techniques measure the bulk flow velocity, while cross-correlation techniques measure the motion of the scatterers in the medium. The UDV is normally used to determine the particle displacements along the longitudinal axis of prefocused transducers. In medical applications the technique is, however, also used to obtain a full 2D image of for, example, blood flow. This is then done by focusing a transducer array in transmit mode, and perform a sequential scan, one sector of the region of interest at a time. In this case, the biological cell displacements are extracted from all the line signals that compose the sectorial scan images. In the human body, the UDV still 
functions because the flow rate in blood vessels is low enough to ensure that two sequential signals of two consecutive sectorial scans-that are generally taken at a frame rate in the range of $50-60 \mathrm{~Hz}$ - are still correlated.

In this paper, a two-dimensional ultrasonic speckle correlation velocimetry technique (USV) [1] is used to measure the particle velocities directly over the entire 2D cross-section of the flow pipe, without the need of any sequential scanning. The technique is associated with an ultrafast ultrasonic imaging system that increases the frame rate of ultrasonic images and allows us to measure significantly higher flow rates than with a traditional phased array system. The USV technique is similar to the UDV technique in the sense that it uses crosscorrelation process to extract the time delay, which in turn gives the particle displacement and velocity. With the USV technique, however, the ultrasonic image is not obtained using a sequential scan over the region of interest, but after only one snapshot. The principle is described in detail in Section 2. The USV technique is especially interesting in the case when the flow pipe is not axi-symmetric or when the flow profile is not axisymmetric, i.e. when a 2D knowledge of the flow velocity is required. Furthermore, the USV technique is a useful complement to conventional laser-Doppler or other optical techniques, since it does not require the flow to be transparent.

For applications where the goal is to measure the mass flow of the solid phase, this technique can be combined with some other method that measures the mass fraction of the solid phase. One example of such a technique is based on the attenuation of pulsed ultrasound [2]. An overview of other techniques can be found in [3].

The USV technique presented here has previously been used in other applications to track particles, or to image vortices in a flow, see for example $[1,4,5]$. In some of these cases, the flow was seeded with a medical contrast agent with density close to that of the liquid. Because of this, tracking the motion of the particles should be a reasonable approximation of the motion of the liquid. In the case of solid/liquid multiphase flows, the use of a contrast agent is not necessary, since the scatterers are naturally present in the flow.

The next section describes the setup used in the experiments, as well as the mathematical principle of the USV technique. The feasibility of the technique for multiphase flow measurements is then examined with experiments on both a medical contrast agent (as a reference measurement) and a suspension of Magnetite (iron ore) and water. The results are presented in Section 3.

\section{Measurement principle}

The measurement of the particle velocity profiles are made using a fast imaging technique, related to tra- ditional phased array imaging used in the medical area, but with some important exceptions that increases the frame rate, allowing us to measure faster moving phenomena.

The following subsections describe the experimental setup, the principle of speckle tracking imaging, and the extension to the ultrafast imaging system used in this paper.

\subsection{Experimental setup}

For the experiments we used a 64-element transducer array with a center frequency of $3.5 \mathrm{mHz}$ and with a $0.417 \mathrm{~mm}$ element pitch. The array was mounted perpendicular to the flow, with an incident angle of $45^{\circ}$ (see Figs. 1 and 2). The flow pipe was vertical, to ensure that even for large density ratios between the solid and liquid phases, the sedimentation would be minimal. The flow meter was mounted after $1 \mathrm{~m}$ of straight pipe, in order to have a fully developed flow profile. The flow pipe was made of PVC plastic. The inner pipe diameter was $33.7 \mathrm{~mm}$. What is not shown in the figure, but is important, is that the flow can be redirected to another container in order to measure a reference volume flow.

From the transducer incident angle $\left(45^{\circ}\right)$ and the particle displacement along the longitudinal axis, $r$, of the transducer array, the particle velocity, $v_{x}$, in the flow direction is given by

$v_{x}=v_{r} \cdot \sqrt{2}$.

Each element of the 64-element transducer array is connected to its own channel of the electronics. Each channel consists of a 9-bit AD/DA converter with a sampling frequency of $30 \mathrm{MHz}$, and $128 \mathrm{MB}$ of memory. This system will be described more in Section 2.3.

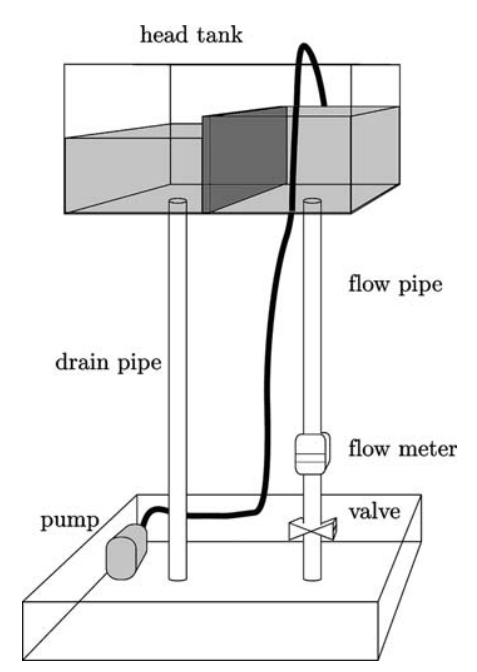

Fig. 1. Setup used in the experiments. The barrier in the head tank ensures constant pressure in the flow pipe. The flow velocity is controlled by the valve at the bottom of the pipe. 


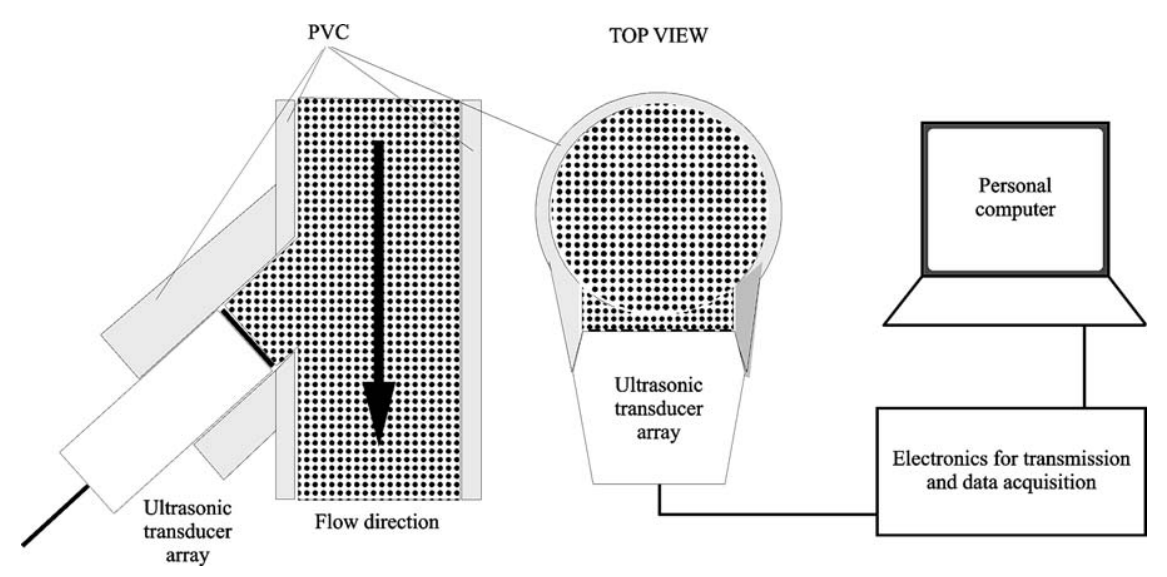

Fig. 2. Close-up of flow meter.

\subsection{Ultrasonic imaging}

In ultrasonic imaging, a transducer array is used to obtain spatial information about the measurement volume. Often the array is focused (beam-formed) in a certain direction, in both transmit mode and receive mode. The full image of the medium is obtained by sequentially scanning all directions. Inhomogeneities in the medium will scatter the sound wave, and the back-scattered sound (received by the array) is used to form the image.

The scatterers in the medium give rise to an interference pattern at the receiver. Reflections from groups of scatterers will arrive at the different transducer elements at different times. Using a receive-mode beamforming technique called parallel beamforming (see Section 2.4), groups of scatterers giving rise to coherent sound waves, can be localized. In speckle correlation imaging, the motion of these groups of scatterers is followed using a cross-correlation technique (Section 2.5).

The lateral spatial resolution of the imaging system depends on $\lambda f / D$, where $\lambda$ is the acoustic wavelength, $f$ the focusing length and $D$ is the size of the transducer aperture. In pulse-echo mode, the spatial resolution along the axial direction depends mainly on the duration of the ultrasonic pulse. Therefore, the position tracking of scatterers from ultrasonic images is trivial if each one can be spatially resolved by the ultrasonic imaging system, according to the above definitions. When the scatterer concentration increases, this will give rise to speckle in the ultrasonic images, due to interference among acoustic waves from the scatterers. In this case, spatial discrimination of individual scatterers is no longer possible.

In the pure diffusion regime, speckle due to a group of scatterers evenly enclosed inside a larger volume, occupies only a limited part of the ultrasonic image. When the group of scatterers moves, the corresponding speckle area in the ultrasonic image is also shifted according to the direction of motion. The other parts remain stationary. Such an analysis is commonly used in ultrasonic medical imaging to observe the flow velocity in blood vessels using the blood cells as scatters. The cross-correlation between two consecutive ultrasonic images is calculated to determine the displacement of the groups of scatterers, and then of the blood flow, according to the axial direction of the imaging system $[6,7]$.

In the medical area, the ultrasonic images obtained with conventional pulse-echo ultrasonic system is achieved using a sequential scanning system, scanning the region of interest in one direction at a time. In this case, the number of images for a time unit (frame rate) is limited to 25-50 images/s. As the flow velocity is low in comparison to the frame rate, the correlation between two successive images is high enough to ensure good velocity estimates. However, when the flow velocity is high, such a system no longer works. The correlation between two consecutive ultrasonic images decreases and the cross-correlation technique leads to erroneous results. For higher flow rates, an imaging system with a higher frame rate is needed.

At Laboratoire Ondes et Acoustique in Paris, an ultrafast ultrasonic imaging system was recently constructed. The initial purpose of such a system is to observe the propagation of low velocity shear waves in soft tissue [8]. In this paper, this fast imaging system is applied to image the 2D particle cross-section velocity profile in multiphase flows. Our purpose is to demonstrate the feasibility of such a technique, and to show that the liquid velocity for some cases is different from the particle velocity.

\subsection{Ultrafast ultrasonic imaging}

The working principle of the ultrafast imaging system is shown in Fig. 3. A plane wave is first used illuminate the medium. This is done by exciting all transducer elements simultaneously. Each transducer element works in pulse-echo mode and is connected to its individual channel of the electronic system. Each channel has its 
(a)

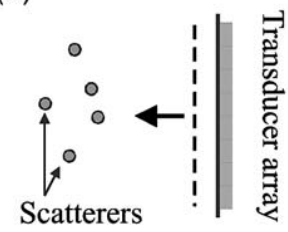

Plane wave (b)

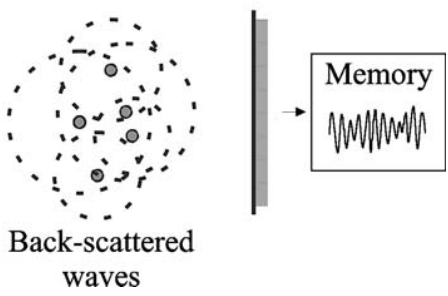

Fig. 3. The imaging sequence. (a) The medium is illuminated using a plane ultrasonic wave. (b) The BS signals are received by the same transducer array and stored in the channel memories.

own AD/DA converter, and a $128 \mathrm{MB}$ memory used to store the backscattered signals (BS) or RF signals. As soon as reception is completed, the transducer array can be used to emit a new plane wave. As the focusing is not performend during the illumination step (i.e. no sequential scanning) the frame rate of such an imaging system can be as high as 10000 frames per second. The number of BS signals that can be captured in a row, depends on their time duration (i.e. the depth of the region of interest), and the available memory in the system.

In our experiment, a linear array composed of 64 transducers with a center frequency of $3.5 \mathrm{mHz}$ was used. The sampling frequency was $30 \mathrm{mHz}$. Since the duration of the BS signal response that must be stored in each channel memory in order to construct a speckle image is typically about $60 \mu \mathrm{s}$ (1800 points), the maximum number of frames that can be stored is 71 .

Once the maximum number of frames is stored, all data are transferred to a PC for additional post-processing. The processing consists of two steps: Parallel beam-forming and speckle correlation.

\subsection{Parallel beam-forming}

The parallel beamforming (PBF) is applied to the BS signals received by each of the 64 transducer elements and stored in electronic memories (Fig. 4a). PBF consists of delaying and adding responses from the different

elements with respect to the abscissa positions in the image. The result of the PBF process is a focused speckle image (Fig. 4b).

The position of the $i$ th element of the array is $(i \Delta x, 0)$, where $\Delta x$ is the width of an element $(\Delta x=$ $0.417 \mathrm{~mm}$ ) and the position of a point $M$ in the medium is $(x, z)$ (Fig. 5). The time of flight of the plane wave to reach point $M$ is equal to $z / c$ where $c$ is the speed of sound. The distance between $M$ and the $i$ th element is

$d_{i}(x, z)=\frac{\sqrt{z^{2}+(x-i \Delta x)^{2}}}{c}$

The time $t_{\mathrm{i}}(x, z)$ needed for the wave to reach $M$, be scattered and go back to the $i$ th element is then

$t_{i}(x, z)=\frac{z+\sqrt{z^{2}+(x-i \Delta x)^{2}}}{c}$

Assuming the plane wave is emitted at time $t=0$, the beamformed BS signals $r_{B F}(x, t)$ computed for the abscissa $x$ is expressed as a function of the BS responses $r_{i}(t)$ stored for each element $(0 \leq i \leq N-1)$. Because of the sampling, the time value $t$ is replaced by its discrete value, $j \Delta t$. The beam-formed RF signals $r_{B F}(x, j \Delta t)$ is then expressed as follows:

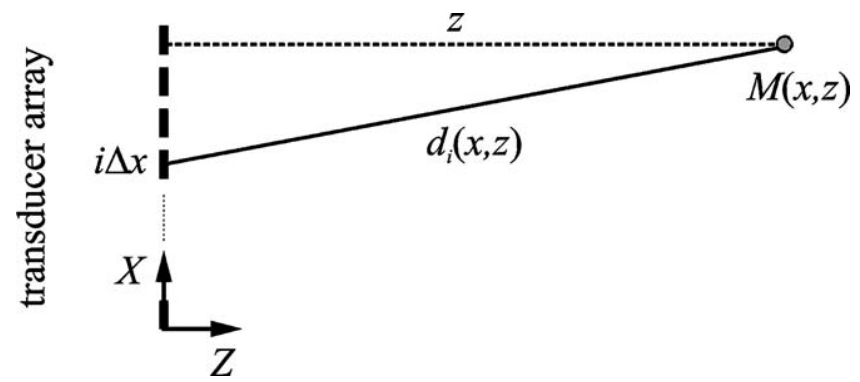

Fig. 5. The time needed for the wave to reach the point $M(x, z)$ is $z / c$, and the time for the wave to travel back to array element $i$ is $d_{i}(x, z) / c$. (a)

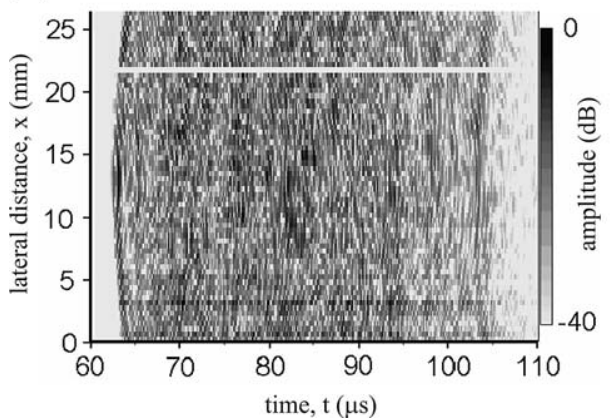

(b)

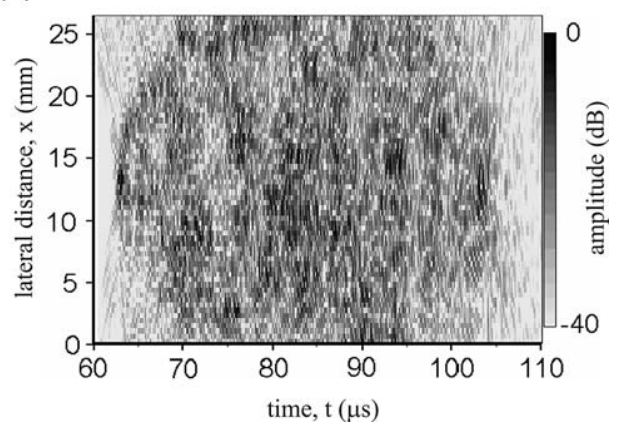

Fig. 4. The parallel beamforming process. (a) $50 \mu$ s long BS signals. (b) Speckle image (BF images) computed by delaying and adding responses from the different transducer elements, with respect to the abscissa positions, $x$, in the image in (a). 


$$
\begin{aligned}
r_{B F}(x, j \Delta t=2 z / c) & = \\
& =\sum_{i=0}^{N-1} r_{i}\left(\frac{j \Delta t}{2}+\sqrt{\left(\frac{j \Delta t}{2}\right)^{2}+\left(\frac{x-i \Delta x}{c}\right)^{2}}\right) .
\end{aligned}
$$

Such an expression corresponds to a continuous dynamic focusing over the full depth, but at discrete values $z=c j \Delta t / 2$. A three-point quadratic interpolation is used to determine amplitude of the BS responses $r_{i}(t)$ at non-discrete time values. Finally the parallel beamformed BS signal $r_{B F}(x, j \Delta t)$ is not only a time-space signal, it is also a space-space amplitude since $z=c j \Delta t / 2$. Fig. 4a shows an example of a $50 \mu$ s long region of the 64-element BS responses, obtained during an experiment on a cylindrical gel sample enclosing micro-marbles. Fig. $4 \mathrm{~b}$ is the corresponding speckle image, computed using the steps described above. The lack of focus during the illumination step affects the dynamic range of speckle images obtained with this technique. The reason is that if the focusing is done in the transmit mode (as in phased array systems), the signal-to-noise ratio (SNR) will be higher, because more energy is transmitted in each direction. High dynamic range is, however, not needed, since we intend to perform cross-correlation between consecutive speckle images. Cross-correlation is more sensitive to phase than to amplitude and is known to give good results even with 1-bit data [9]. The $\mathrm{PBF}$ is performed on a PC and it takes a few seconds per frame. The total computation time can be up to a couple of minutes, depending on how many frames we choose to store in the memory of the imaging system before doing the ofliine calculations.

\subsection{Axial displacement estimation}

Many speckle tracking algorithms [7] have been developed in order to estimate the axial or even lateral displacement or strain in soft tissue. In our study, the speckle lines are segmented vs. depth $z$ in 1-mm slices. The axial displacement is estimated in each of the segments using the cross-correlation technique [10] between successive speckle images. The resolution of the axial displacement estimates is about $1 \mu \mathrm{m}$ [11].

Table 1 shows the full process used to obtain the particle displacement images. After PBF of $N_{F}$ unfocalized speckle data set, $N_{F}$ speckle images are obtained. Plane waves are periodically emitted at a frequency corresponding to the frame rate $1 / T_{p r f}$. The speckle images are described by $r_{B F}(x, z=c j \Delta t / 2)$ with the beam-formed BS signals.

\section{Experiments}

To evaluate the technique we prepared two different suspensions, with different density ratios between the solid and the liquid phase. The first suspension was water and the medical contrast agent Sonazoid. ${ }^{1}$ This contrast agent consist of small micro bubbles with approximately the same density as water. The second suspension was Magnetite $\left(\mathrm{Fe}_{3} \mathrm{O}_{4}\right)$ particles and water. The density of the Magnetite was $5200 \mathrm{~kg} / \mathrm{m}^{3}$, which is significantly higher than that of water.

\section{Results}

Figs. 6 and 8 show the particle velocity cross-sections of Sonazoid and Magnetite, respectively, for two different velocities. Figs. 7 and 9 show the velocity profiles at the center transducer element of the array, for five different velocities. A hole was cut in the wall of the pipe at the position where the transducer array was mounted (see Fig. 2). At this position small vortices formed, which disturbed the otherwise axi-symmetric flow profile. This can be seen at the bottom of Figs. 6 and 8, but even clearer on the left side of Figs. 7 and 9. Since the average particle velocity is calculated over the entire 2D flow profile, this should not, however, affect the estimation of the particle velocity.

For each of the experiments a measurement of the liquid volume flow was also made. The liquid volume flow velocities were compared with the average particle velocities, taken over the whole flow profiles in Figs. 6 and 8 . The volume flow was determined by measuring the amount of liquid that falls through the right pipe (see Fig. 1) into the bottom tank during a short time period. The velocity is deduced from the volume flow and the inner cross section area of the PVC pipe. This comparison is shown in Fig. 10. The comparison shows that for the contrast agent, where the density of the particles is approximately the same as that of the liquid, the two methods give similar results. For the Magnetite measurements we note that the measured particle velocities are in average higher than the reference flow, because the particles are heavier than the liquid, and because of the vertical flow setup (moving downwards). The difference in flow velocities is therefore expected and depends on factors such as liquid viscosity, particle sizes, and solid/liquid density ratio [12].

\section{Discussion}

The measurement principle presented in this paper enables us to measure displacements along the trans-

\footnotetext{
${ }^{1}$ Sonazoid is a registered trademark of Nycomed Amersham A/S.
} 
Table 1

From $N_{F}$ BS data sets, $N_{F}$ speckle images are obtained, which lead to $N_{F}-1$ displacement images. Speckle data sets are obtained at a frame rate equal to $1 / T_{p r f}$

\begin{tabular}{|c|c|c|c|c|c|c|}
\hline Frame no. & & $r_{i}(j \Delta t)$ & & $r_{B F}(x, z=c j \Delta t / 2)$ & & Displacements \\
\hline 0 & $\rightarrow$ & BS echoes 0 & $\rightarrow$ & Speckle image 0 & & \\
\hline 1 & $\rightarrow$ & BS echoes 1 & $\rightarrow$ & Speckle image 1 & $\rightarrow$ & Image 0 \\
\hline 2 & $\rightarrow$ & BS echoes 2 & $\rightarrow$ & Speckle image 2 & $\rightarrow$ & Image 1 \\
\hline$\vdots$ & & $\vdots$ & & $\vdots$ & & $\vdots$ \\
\hline$N_{F}-1$ & $\rightarrow$ & BS echoes $N_{F}-1$ & $\rightarrow$ & Speckle image $N_{F}-1$ & $\rightarrow$ & Image $N_{F}-2$ \\
\hline
\end{tabular}

(a)

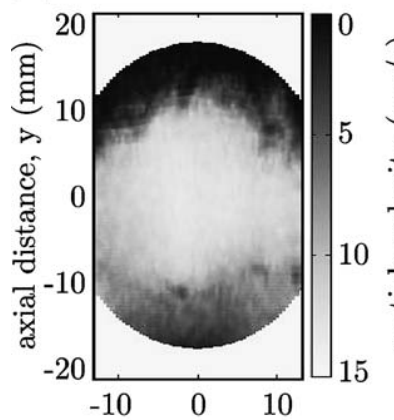

transversal distance, $\mathrm{x}(\mathrm{mm})$ (b)

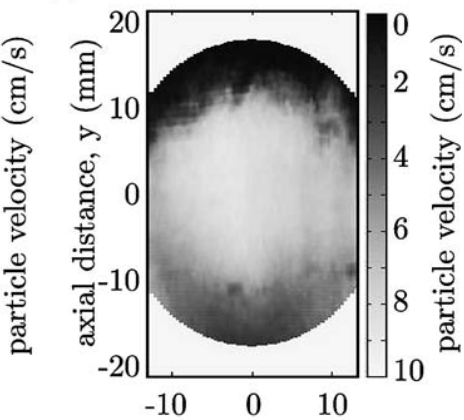

transversal distance, $\mathrm{x}(\mathrm{mm})$

Fig. 6. Sonazoid particles velocity profiles for entire cross section of the flow. (a) Volume flow velocity, $8.96 \mathrm{~cm} / \mathrm{s}$. (b) Volume flow velocity, $5.72 \mathrm{~cm} / \mathrm{s}$. The array was located at the bottom of the images.

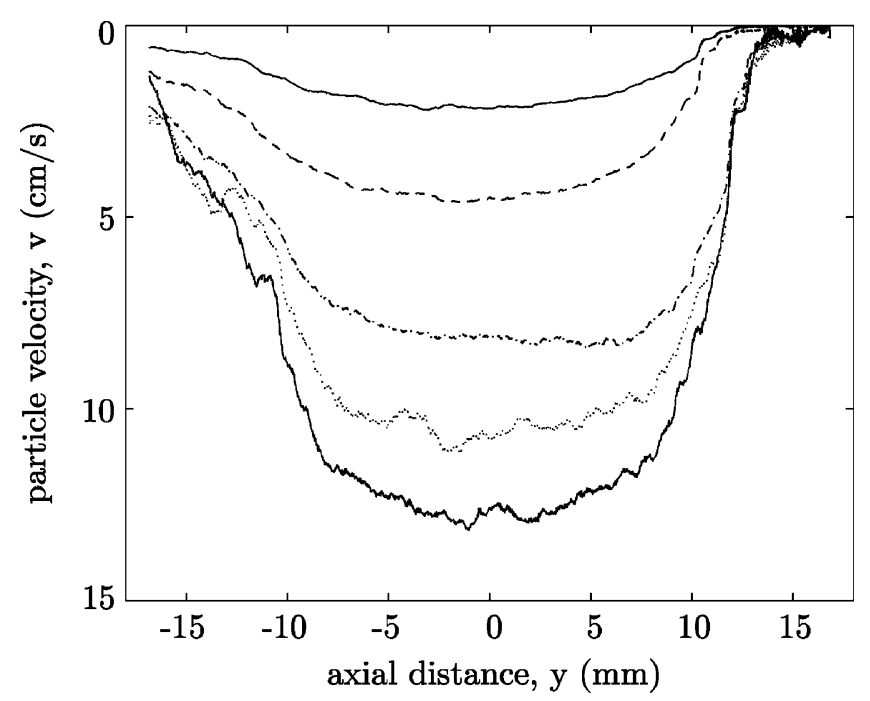

Fig. 7. Velocity profiles for Sonazoid at the center transducer element, corresponding to five values of the volume flow velocity (from top to bottom in the figure): $1.49 \mathrm{~cm} / \mathrm{s}, 2.96 \mathrm{~cm} / \mathrm{s}, 5.72 \mathrm{~cm} / \mathrm{s}$, $7.93 \mathrm{~cm} / \mathrm{s}$ and $8.96 \mathrm{~cm} / \mathrm{s}$. The hole in the pipe, where the array was mounted, was to the left in the plot.

ducer axis. This can be done over the entire cross-section of the pipe, which is a major improvement compared to single-transducer Doppler- or correlation-based techniques. Transversal displacements, however, cannot be estimated with the proposed method. The reason for this

(a)

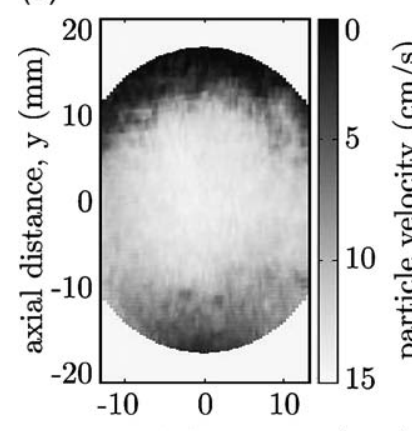

(b)

transversal distance, $\mathrm{x}(\mathrm{mm})$

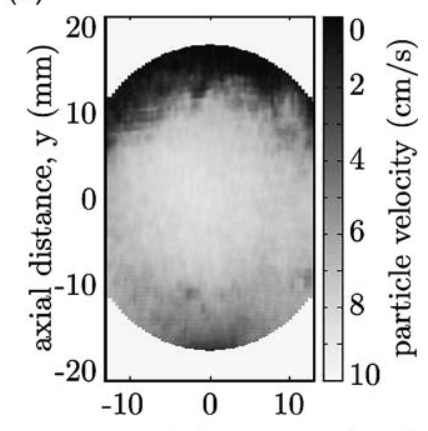

Fig. 8. Magnetite particles velocity profiles for entire cross section of the flow. (a) Volume flow velocity, $8.96 \mathrm{~cm} / \mathrm{s}$. (b) Volume flow velocity, $5.72 \mathrm{~cm} / \mathrm{s}$. The array was located at the bottom of the images.

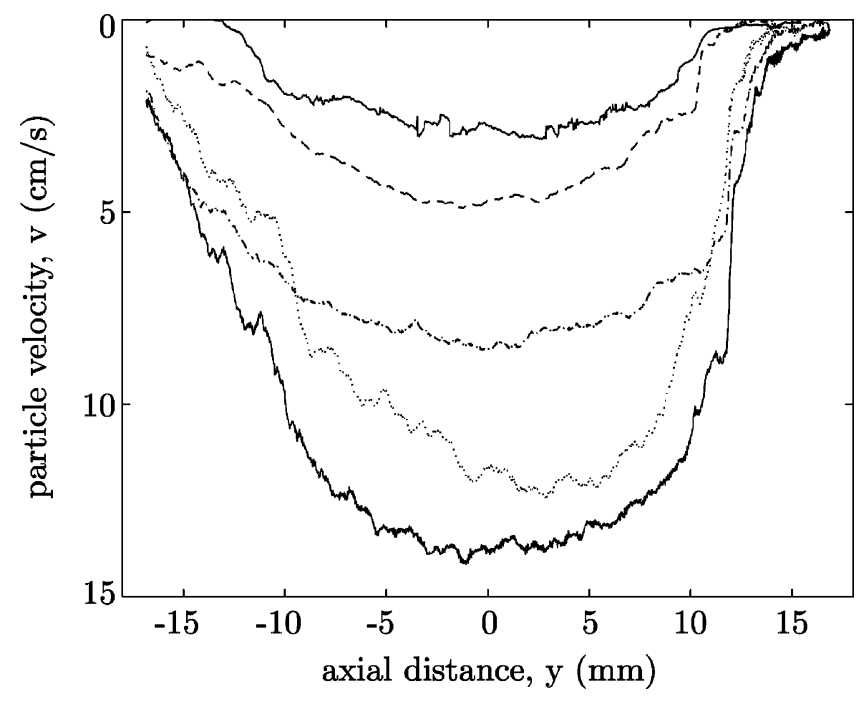

Fig. 9. Velocity profiles for Magnetite at the center transducer element, corresponding to five values of the volume flow velocity (from top to bottom in the figure): $1.49 \mathrm{~cm} / \mathrm{s}, 2.96 \mathrm{~cm} / \mathrm{s}, 5.72 \mathrm{~cm} / \mathrm{s}$, $7.93 \mathrm{~cm} / \mathrm{s}$ and $8.96 \mathrm{~cm} / \mathrm{s}$. The hole in the pipe, where the array was mounted, was to the left in the plot.

is that there is a large difference in resolution between the axial and the transversal direction. In the axial direction, the resolution is given by the wavelength of the sound, and the sampling time in the hardware. The measured displacements are in the range of one to two 


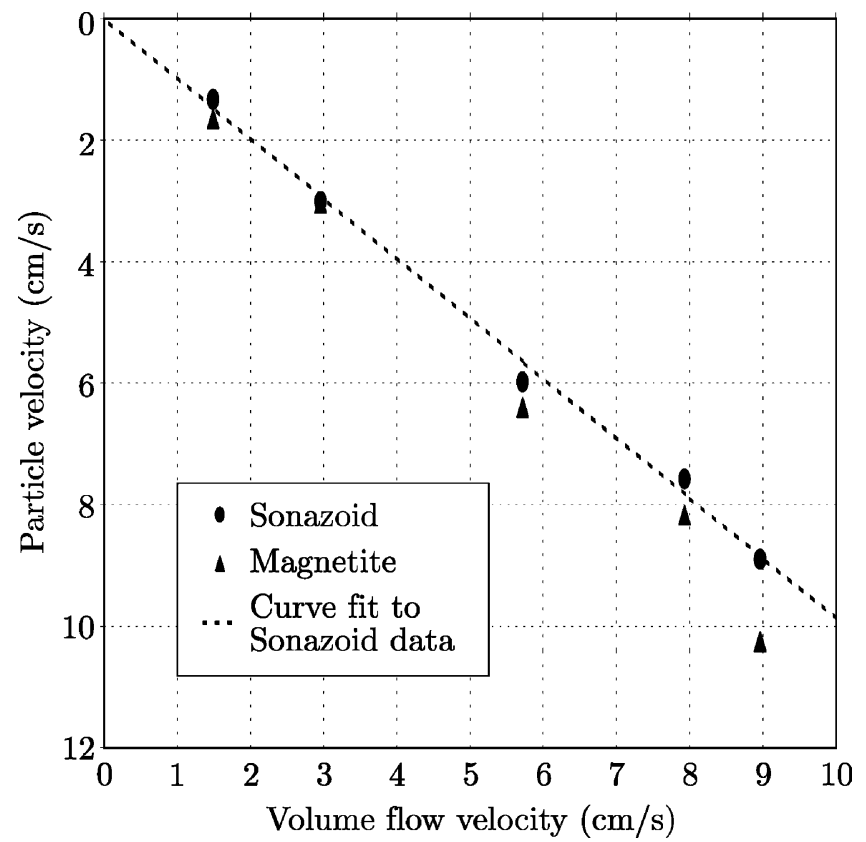

Fig. 10. Comparison with average particle velocity and total volume flow.

samples, which in the current setup corresponds to approximately $0.05 \mathrm{~mm}$. The resolution in the transversal direction, on the other hand, is given by the array pitch (i.e. spacing between array elements.), which is in our case was $0.417 \mathrm{~mm}$. With the current frame-rate, the medium will not move enough for the transversal displacement to be detectable, and if the frame-rate would be lowered for this to change, the ultrasound signals in the axial direction would be decorrelated. In a flow measurement application, the most interesting motion is, however, in one direction and thus the proposed technique gives satisfying results.

In the present setup, there is a small hole cut in the pipe where the transducer array is mounted. This causes the measured flow profile to be non-symmetrical, because small vortices are formed around the opening. Since the particle velocity is measured as the average over the entire image, this should not, however, affect the accuracy to any significant extent. In a more refined setup, the opening in the pipe should be replaced with an acoustically transparent film.

\section{Conclusions}

In this paper, we show how 2D USV can be used to measure particle velocity profiles in multiphase flows. This is an important step forward in flow measurement applications where the goal is to measure mass flow of the individual constituent phases. Where traditional Doppler and cross-correlation techniques only measure the particle velocities along the axis of the transducer, the USV technique enables us to measure the particle velocity profile of an entire cross-section of the flow.

The reference measurements of the true volume flow agrees well with the average of the measured particle velocity profile of the medical contrast agent Sonazoid. Because the contrast agent is designed to follow the liquid flow, this is taken as an evidence that the speckle tracking method works well.

For the Magnetite suspension, the measured particle velocities are slightly higher than the reference volume flow. This is, however, an expected result, because in the current experimental setup, all measurements are made on flow moving downwards in a vertical pipe. Since the Magnetite particles are about five times heavier than the liquid, it is reasonable that they move slightly faster than the liquid phase.

\section{Acknowledgements}

This work was made possible by grants from The Research Council of Norrbotten. The authors would also like to express their gratitude towards professor Mathias Fink and professor Jerker Delsing for supporting this work, and to Mr. Michel Parise for his help with the experimental setup. The authors also thank Dr. Jenny Ostensen at Nycomed Amersham A/S for providing the Sonazoid contrast agent.

\section{References}

[1] L. Sandrin, S. Manneville, M. Fink, Ultrafast two-dimensional speckle velocimetry: a tool in flow imaging, Applied Physics Letters 78 (8) (2001) 1155-1157.

[2] J. Carlson, Joint measurement of particle distribution and particle mass fraction in multiphase flows, in: Proc Flow Measurement 2001, Peebles, Scotland, UK, 2001.

[3] J. Chaoki, F. Larachi, M.P. Dudukovic (Eds.), Non-Invasive Monitoring of Multiphase Flows, Elsevier, Amsterdam, The Netherlands, 1997.

[4] S. Manneville, L. Sandrin, M. Fink, Investigating a stretched vortex with ultrafast $2 \mathrm{~d}$ ultrasonic speckle velocimetry, Physics of Fluids 13 (6) (2001).

[5] J. Carlson, R.K. Ing, J. Bercoff, M. Tanter, Vortex imaging using two-dimensional ultrasonic speckle correlation, in: Proc of IEEE Int Ultrason Symp, vol 1, Atlanta, GA, USA, October, 2001, 2001, pp. 559-562.

[6] S. Catheline, F. Wu, M. Fink, A solution to diffraction biases in sonoelasticity: the acoustic impulse technique, J. Acoust. Soc. Am. 105 (1999) 2941-2950.

[7] M. O’Donnell, A.R. Skovoroda, B.M. Shapo, Y. Emelianov, Internal displacement and strain imaging using ultrasonic speckle tracking, in: IEEE Trans Ultrason, Ferroelec and Freq Contr, vol. 41, 1994, pp. 314-325.

[8] L. Sandrin, S. Catheline, M. Tanter, X. Hennequin, and M. Fink, Time-Resolved Pulsed Elastography with Ultrafast Ultrasonic Imaging, Ultrasonic Imaging, 2002. Submitted.

[9] O. Bonnefous, P. Pesque, A new velocity estimator for color flow mapping, in: Proc IEEE Int Ultrason Symp, 1986, pp. 855-860. 
[10] J. Jensen, Estimation of Blood Velocities Using Ultrasound, Cambridge University Press, 1996 Ch 8.

[11] W.F. Walker, G.E. Trahey, A fundamental limit on the performance of correlation based on phase correction and flow estimation technique, in: IEEE Trans Ultrason, Ferroelec and Freq Contr, vol 41, 1994, pp. 644-654.

[12] G.K. Batchelor, Introduction to Fluid Dynamics, Cambridge University Press, Cambridge, UK, 1967 p. 234. 